\title{
Distribution of Mating Type and Sexual Status in Chinese Rice Blast Populations
}

\author{
Jing Zeng, Shujie Feng, Jiangqiao Cai, Ling Wang, Fei Lin, and Qinghua Pan, Laboratory of Plant Resistance \\ and Genetics, College of Natural Resources and Environmental Science, South China Agricultural University, \\ Guangzhou, 510642, China
}

\begin{abstract}
Zeng, J., Feng, S., Cai, J., Wang, L., Lin, F., and Pan, Q. 2009. Distribution of mating type and sexual status in Chinese rice blast populations. Plant Dis. 93:238-242.

A collection of 520 field isolates of the rice blast fungus (Magnaporthe oryzae) originating from five provinces in China was assessed for mating type and sexual fertility. One of the two tester sets was composed of isolates collected from barley and the other from rice. Two mating types (MAT1-1 and MAT1-2) were identified among the 443 fertile isolates. The two mating types were roughly in balance with one another in the southwestern region but one or the other predominated in the southeastern and southern regions. Male-only fertile isolates were the most common, and only a few hermaphroditic and no female only fertile isolates were detected. The fertility level of the isolates was variable. Isolates from Jiangsu were more fertile than those from Fujian. The mating capacity of the testers collected from barley was higher than that of those collected from rice, but this was because the MAT1-2 testers differed very significantly from one another. In contrast, the mating capacities of the two MAT1-1 testers were similar to one another.
\end{abstract}

Magnaporthe oryzae B.C. Couch is the causal organism of rice blast, one of the most severe fungal diseases of rice worldwide $(3,12,16,24)$. In addition to its infecting rice (Oryza sativa L.), M. oryzae also is pathogenic on approximately 50 other grass species, including a number of economically important crops such as barley, wheat, and millet $(2,27)$. The major determinants of $M$. oryzae population genetic structure and its variation over time and space are mutation, resistance gene $(R$ gene)-driven selection, gene flow between populations, parasexual recombination within isolates, and genetic recombination between isolates $(17,20,27)$. The latter possibility remains theoretical because, to date, sexual reproduction of $M$. oryzae in the field has not been observed $(2,7,8,12,16,27)$.

As for other heterothallic ascomycetes, sexual compatibility in $M$. oryzae is determined by the presence of two idiomorphs at a single mating-type locus, designated MAT1-1 and MAT1-2. For mating and sexual reproduction to take place, both mating types must be present $(2,13,18,20$ 22,27 ). However, one mating type usually

Corresponding author: Q. Pan

E-mail: panqh@scau.edu.cn

J. Zeng and S. Feng contributed equally to this work.

Current address of S. Feng: Laboratory of Plant Protection, College of Horticulture, South China Agricultural University, Guangzhou, 510642, China.

Accepted for publication 20 November 2008.

doi:10.1094/PDIS-93-3-0238

(c) 2009 The American Phytopathological Society dominates in natural $M$. oryzae populations $(1,2,18,27)$, and this highly skewed mating type ratio is believed to be a major block to sexual recombination under field conditions $(2,16,21)$. Even where both mating type isolates coexist, other barriers, in particular the sexual and fertility status of the fungus, also can hinder successful mating $(13,21,22)$. The sexual status of $M$. oryzae isolates can be classified as hermaphroditic (male and female fertile), female fertile but male sterile, male fertile but female sterile, and sterile $(22,27)$, with most isolates being either male-only fertile or completely sterile $(9,10,16)$. In the past, it has only been possible to obtain crosses between a small number of $M$. oryzae isolates because of the rarity of female fertile or hermaphroditic isolates $(10,19)$. More recently, however, the identification of a number of hermaphroditic isolates has allowed many more controlled crosses to be made $(5-8,14,15,26)$. However, even where perithecia are produced, both their fertility and the viability of the ascospores within them are poor $(10,18)$.

The genetic dissection of pathogen traits requires a degree of fertility to generate a sufficiently large progeny population $(6,18,24)$. Low fertility may also reflect some incompatibility between the tester and field isolates $(11,16)$. Because rice $M$. oryzae isolates usually are not fertile, it has been suggested that crosses made between a rice isolate and one pathogenic on other Gramineae plants could help to determine the fertility status of the rice blast isolates (19). That is, the fertility of isolates would vary if the tester isolates derived from different hosts. Thus, for a proper investigation of the sexual and fertility status of a rice blast population, it is important to work with a range of different tester isolates $(1,25)$.

The distribution of mating type has been used to characterize the genetic structure and dynamics of heterothallic populations $(2,16,18,21,22,27)$. Because the presence of female-only-fertile or hermaphroditic isolates in a population suggests that sexual reproduction has probably occurred in the recent past, the likelihood that sexual reproduction will be a factor in the future is heightened $(20,22)$. An understanding of both the mating type distribution and the sexual and fertility status of an M. oryzae population can provide insights into its evolutionary history $(2,20,21)$. In this study, we have targeted $M$. oryzae populations collected from three ecosystems in China. Our objectives were to investigate their mating type distribution and sexual status, and compare the relative mating capacities between pairs of tester isolates derived from various plant hosts.

\section{MATERIALS AND METHODS}

Isolates collection and maintenance. Plants with typical blast symptoms on their panicles were sampled during 2001-03 from rice fields across Fujian (FJ), Guangdong (GD), Yunnan (YN), Guizhou (GZ), and Jiangsu (JS) provinces, China. These areas are representative of the three distinct rice-growing ecosystems within China (Fig. 1). Each panicle sample was surface disinfected with $70 \%$ ethanol and laid on a sheet of sterile wetted tissue paper in a $9-\mathrm{cm}$ petri dish. After $24 \mathrm{~h}$ of incubation at $25^{\circ} \mathrm{C}$, single spores were collected on the tip of a glass pin under a stereomicroscope, and cultured on a potato dextrose agar (PDA; potato at $200 \mathrm{~g} \mathrm{liter}^{-1}$, dextrose at $20 \mathrm{~g} \mathrm{liter}^{-1}$, and agar at $15 \mathrm{~g}$ liter $^{-1}$ ) slope. This generated a set of 520 monoconidial isolates (Table 1). To avoid genetic drift via mutation during culture and to allow for isolate multiplication, each isolate was transferred to long-term storage, using the filter-paper-based method described elsewhere (6).

Tester isolates and crossing. Two sets of standard isolates were used for testing mating type. Set A consisted of isolates TH12 (MAT1-1) and TH16 (MAT1-2) (both collected from barley in Thailand), and set B consisted of CHL43 (MAT1-1) and CHL42 (MAT1-2) (isolated from rice in Yunnan). All of these isolates are hermaphroditic and produce many perithecia. Crosses were made by a four-point culture method, in which two field isolates and 
one pair of tester isolates were placed symmetrically about $3 \mathrm{~cm}$ apart on oatmeal agar medium (oatmeal at $50 \mathrm{~g} \mathrm{liter}^{-1}$ and agar at $12 \mathrm{~g} \mathrm{liter}^{-1}$ ) in a 9-cm petri dish. The plates were incubated at $25^{\circ} \mathrm{C}$ for 5 to 7 days, until the mycelia of the two paired individuals merged $(1,2)$. The cultures were then transferred to continuous white fluorescent light at $20^{\circ} \mathrm{C}$. After 15 to 20 days, mature perithecia formed at the intersection of growth between mated individuals were evaluated.

Assays for mating type and sexual and fertile status. The mating type of the field isolates was determined in reciprocal crosses (16), so that TH12 and CHL43 were used to identify MAT1-2 and TH16 and CHL42 to identify MAT1-1. The mat- ing type of isolates that failed to mate with any of the four testers was designated "not determined" (ND). The assignment of sexual status followed the established nomenclature $(9,16)$, whereby male-onlyfertile isolates form perithecia on the tester isolate side of the merged zone whereas, for female-only-fertile ones, perithecia are formed on the field isolate side. Field iso-

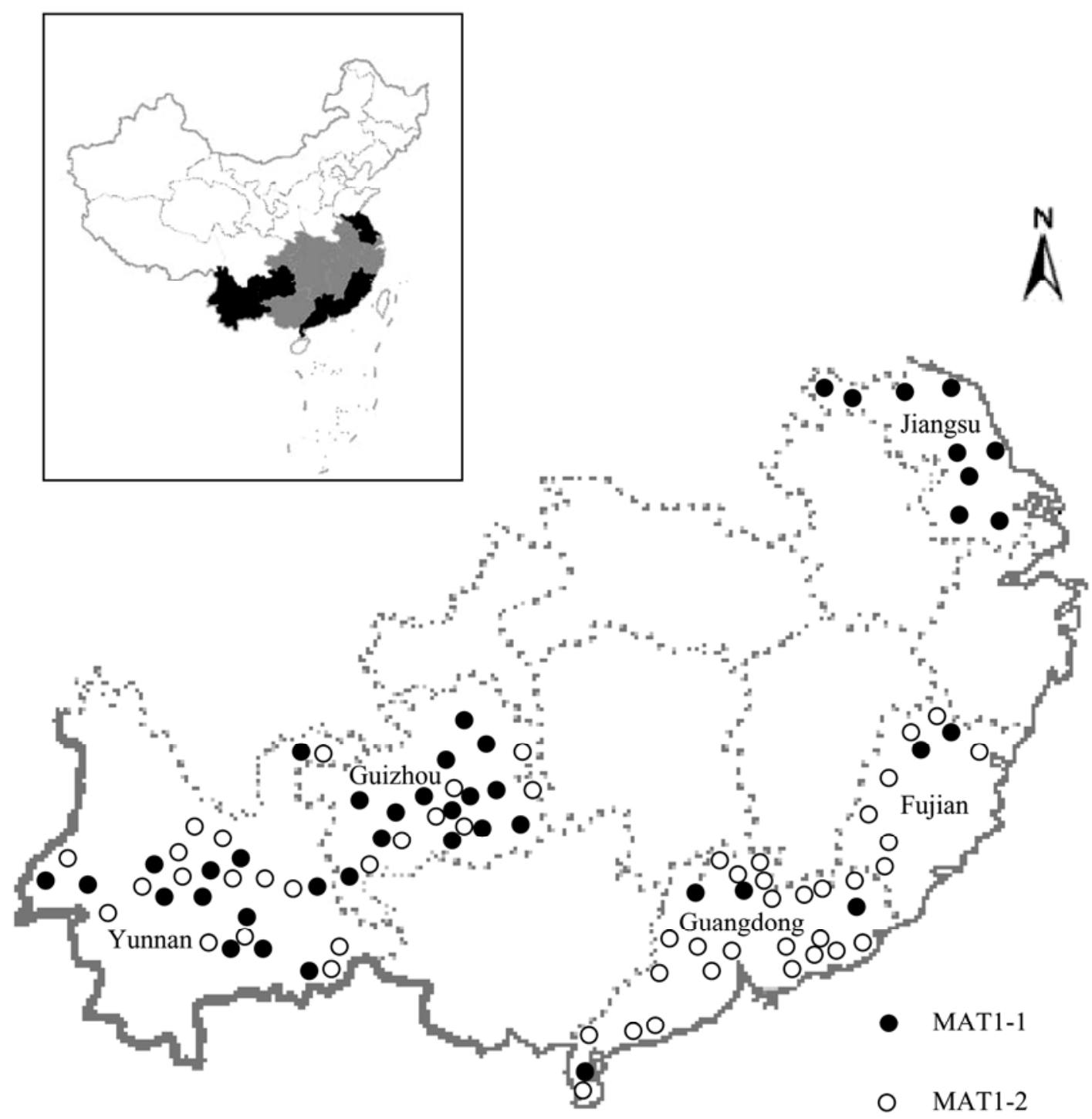

Fig. 1. Sampling locations of field Magnaporthe oryzae isolates from Fujian, Guangdong, Yunnan, Guizhou, and Jiangsu provinces, representing the three major rice ecosystems in China.

Table 1. Geographical distribution of isolates involved in successful matings and a least significant difference test comparison of mating capacity between two tester isolate sets in five populations of Chinese Magnaporthe oryzae ${ }^{\mathrm{y}}$

\begin{tabular}{|c|c|c|c|c|c|c|c|c|c|c|}
\hline \multirow[b]{2}{*}{ Population } & \multirow[b]{2}{*}{ Size } & \multicolumn{4}{|c|}{ Set A } & \multicolumn{4}{|c|}{ Set $B$} & \multirow[b]{2}{*}{$F^{A+B}(\%)^{z}$} \\
\hline & & TH12 (MAT1-1) & TH16 (MAT1-2) & ND & $F^{A}(\%)$ & $\overline{\text { CHL43 (MAT1-1) }}$ & CHL42 (MAT1-2) & ND & $F^{B}(\%)$ & \\
\hline Fujian & 77 & 67 & 3 & 7 & $90.9 \mathrm{~A}$ & 12 & 3 & 62 & $19.5 \mathrm{~B}$ & 90.9 \\
\hline Guangdong & 162 & 124 & 12 & 26 & $84.5 \mathrm{~A}$ & 30 & 8 & 124 & $23.4 \mathrm{~B}$ & 85.8 \\
\hline Yunnan & 109 & 39 & 46 & 24 & $78.0 \mathrm{~A}$ & 28 & 41 & 40 & $63.3 \mathrm{~B}$ & 83.5 \\
\hline Guizhou & 95 & 21 & 45 & 29 & $69.5 \mathrm{~A}$ & 7 & 42 & 46 & $51.6 \mathrm{~B}$ & 72.6 \\
\hline Jiangsu & 77 & 0 & 73 & 4 & $94.8 \mathrm{~A}$ & 0 & 67 & 10 & $87.0 \mathrm{~B}$ & 96.1 \\
\hline Total & 520 & 251 & 179 & 90 & $82.7 \mathrm{~A}$ & 77 & 161 & 282 & $45.8 \mathrm{~B}$ & $\ldots$ \\
\hline
\end{tabular}

${ }^{y}$ Set A comprised TH12 and TH16, isolated from barley, while the Set B members (CHL43 and CHL42) were isolated from rice. ND = number of isolates failing to mate with any of the two tester isolates in sets $\mathrm{A}$ and $\mathrm{B} ; F^{A}$ and $F^{B}=$ frequency of mating isolates (equally frequency of fertile isolates) determined by set A and set B of tester isolates, respectively. Values within a row followed by a different letter differ significantly from one another $(P=0.01)$.

${ }^{\mathrm{z}}$ Integrated frequency of mating isolates (equally integrated frequency of fertile isolates) as determined by two sets of tester isolates. 
lates in which perithecia were formed on both sides of the merged zone were designated as hermaphroditic, and the sexual status of those in which the perithecia were tightly restricted to the boundary line was considered to be unknown. The degree of fertility was assessed by the number of perithecia per square centimeter of medium surface $(16,22)$. From each cross, the perithecia were assessed under a stereomicroscope for the presence or absence of ascospores. Isolates yielding $>20$ perithe$\mathrm{cia} / \mathrm{cm}^{2}$ and containing viable ascospores were designated as highly fertile. Isolates yielding 10 to 19 and $<10$ perithecia $/ \mathrm{cm}^{2}$ with viable ascospores were designated as intermediate and poorly fertile, respectively. Two independent replicates of each cross at different times were analyzed.

When a field isolate was typed by one set of testers as being hermaphroditic but by the other as being either only-male or only-female fertile, then the isolate was recorded as being hermaphroditic. Similarly, the fertility of each field isolate was determined by whichever tester set gave the higher estimate.

Data analysis. The frequency of mating isolates in individual populations was calculated as $F^{A}=n^{A} / n$ and $F^{B}=n^{B} / n$, where $n$ represents the total number of isolates tested and $n^{A}$ and $n^{B}$ the numbers of the field isolates identified as fertile by set $\mathrm{A}$ and set $\mathrm{B}$, respectively (Table 1 ). To more precisely estimate the frequency of fertile isolates in the respective populations, two frequencies were combined as $F^{A+B}=\left(n^{A}+\right.$ $\left.n^{B}+n^{C}\right) / n$, where $n^{C}$ is the number of isolates detected by both tester sets (1).

The mating capacities of the two tester sets were evaluated as the frequencies $F^{A}$ and $F^{B}$, respectively, in individual populations. A least significant difference test was applied to compare the mating capacities between tester sets both within and across each of the five populations (Table 1).

\section{RESULTS}

Mating type distribution. The mating type of each of the 520 rice blast isolates is

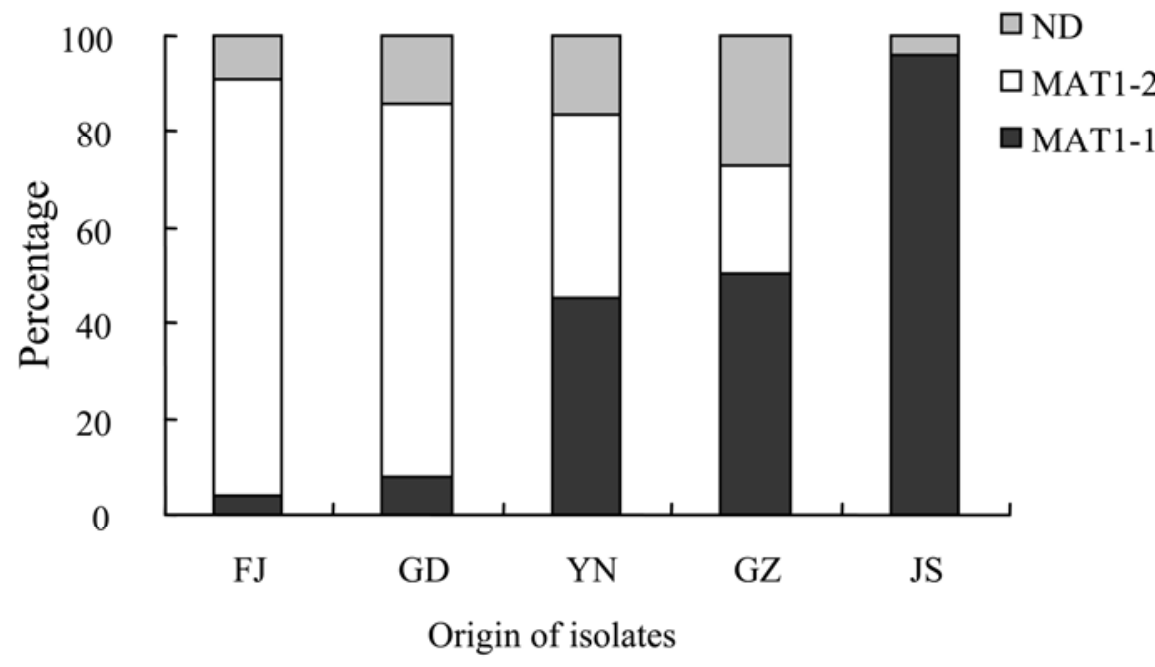

Fig. 2. Distribution of mating types in Chinese Magnaporthe oryzae populations. FJ: Fujian, 77 isolates; GD: Guangdong, 162 isolates; YN: Yunnan, 109 isolates; GZ: Guizhou, 95 isolates; JS: Jiangsu, 77 isolates; ND: mating type not determined, due to failure to mate with any of the four testers.

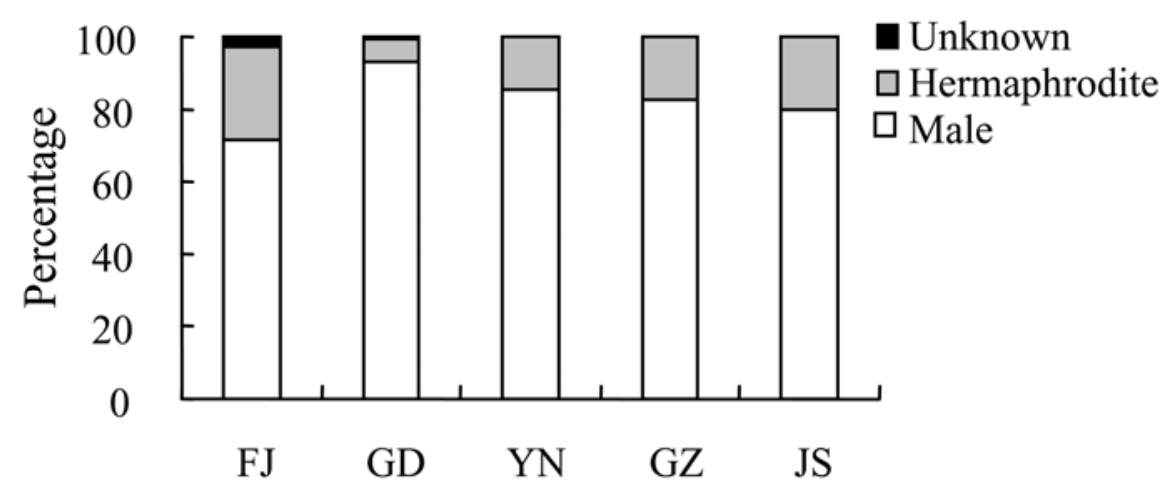

Origin of isolates

Fig. 3. Sexual status among 443 fertile rice blast isolates. FJ: Fujian, 70 isolates; GD: Guangdong, 139 isolates; YN: Yunnan, 91 isolates; GZ: Guizhou, 69 isolates; JS: Jiangsu, 74 isolates. Unknown sex refers to isolates which formed perithecia on the boundary between the tester and field isolates. summarized in Table 1, and the geographical distribution of fertile isolates is depicted in Figures 1 and 2. MAT1-2 isolates predominated in both the FJ and GD populations (southern region). Only MAT1-1 isolates were found in the JS population (southeastern region). Both mating types were frequent in both the YN and GZ populations (southwestern region). In the $\mathrm{YN}$ population, the MAT1-1:MAT1-2 ratio did not differ from $1: 1\left(\chi_{1 \mathrm{df}}^{2}=0.54, P>0.05\right)$.

Fertility and sexual status. The JS population had the highest frequency of fertile isolates $(96.1 \%)$, followed by $\mathrm{FJ}$ (90.9\%), GD (85.8\%), and YN (83.5\%), with the lowest proportion $(72.6 \%)$ present in GZ (Table 1). Of the 443 fertile isolates detected by the two tester sets, male-only fertility was the most common state in all five populations (Fig. 3). No female-only fertile isolates were detected in any of the populations. The proportion of hermaphroditic isolates ranged from $5.8 \%$ (GD) to $25.7 \%$ (FJ). A small number of unassigned isolates was present in GD and FJ. The proportion of highly fertile isolates was highest in the JS (62.3\% of the fertile isolates) and lowest in the FJ $(15.6 \%)$ population. Intermediate fertility was commonest in FJ (75.5\%) and rarest in GZ (26.3\%), while low fertility predominated in GZ $(27.4 \%)$ and was rarest in JS (3.9\%) (Fig. 4).

Mating capacity. Except for the FJ population, the integrated frequency $\left(F^{A+B}\right)$ of fertile isolates for four of the populations (GD, YN, GZ, and JS) was higher than that defined by either set $\mathrm{A}\left(F^{A}\right)$ or set $\mathrm{B}\left(F^{B}\right)$ alone, meaning that the use of two sets of tester isolates instead of one set allowed more isolates to be identified (Table 1). As revealed by the least significant difference analysis, the mating capacity of the set A testers was higher $(82.7 \%)$ than that of the set B testers (45.8\%), especially in the FJ and GD populations. This difference was largely due to the differential abilities of the MATI-1 testers TH12 and CHL43, because TH12 identified 251 isolates while CHL43 identified only 77 (Table 1).

\section{DISCUSSION}

A strong level of variation appears to be associated with the geographical distribution of the two mating types. They were in approximate balance in the southwestern region but either one or the other very much predominated in the southeastern and southern regions. Therefore, it seems plausible that host cultivation or environmental conditions can influence matingtype distribution. This suggestion is not consistent with much of the literature $(2,16,22)$; therefore, its validation will require supportive data from further sampling of Chinese $M$. oryzae isolates. The YN and GZ populations originate from a region of southwestern China which spans the tropical, subtropical, temperate, and 
cold zones. In addition to this varied ecosystem, many rice landraces are commonly cultivated using traditional growing practices $(4,28)$, with as many as 5,000 landraces having been documented in Yunnan province alone (4). The variation in ecosystem and the high level of host diversity could lead to more variation in sexual status (especially female fertility) in populations than what occurs in more uniform environments (13). Hayashi et al. (7) concluded that fertile isolates of $M$. oryzae probably first evolved in southwestern China, and the distribution of fertility found in our survey is consistent with their conclusions.

In surveys of mating type and the sexual and fertility status of pathogen populations, it is important that the tester isolates selected are highly fertile, hermaphroditic, and of known mating type $(1,18)$. The mating capacity of $M$. oryzae isolated from hosts other than rice is thought to be generally higher than when isolated from rice $(1,18,19,23,25)$. Of the two tester sets used in the present experiments, one comprised isolates isolated from barley and the other from rice, and the frequency of fertile isolates in test crosses involving the former was significantly higher than in those involving the latter (Table 1). However, the mating capacities of the two MATl-2 testers (TH16 and CHL42) were similar, whereas those of the MAT1-1 testers (TH12 and CHL43) were substantially different. It should be possible, therefore, to identify $M$. oryzae isolates from rice itself which have a mating capacity as high as CHL42 or the isolate Guy11 used by Notteghem and Silué (18). This is one of the reasons why a decision was made to focus on field isolates as a source of testers in the present study. A field isolate to act as a MAT1-1 tester is needed because both the highly fertile and hermaphroditic isolates CHL42 and Guy11 are MAT1-2 testers. In parallel, we are in the process of breeding tester isolates with higher fertility to use as mating testers, adopting a backcross approach as described in some previous reports $(19,24)$.

Notably, the fertile isolates used to date for making crosses have all originated from southern China, except for Guy11, which may have been introduced to French Guyana via seed exchange (19). Selections from among highly fertile $\mathrm{YN}$ isolates have been used by a number of workers as crossing parents to construct the segregating populations necessary for genetic analysis, gene tagging, and map-based cloning in rice blast $(14,17,26)$. According to results from the present study, we have constructed several progeny populations derived from crosses using CHL42 or CHL43 as parental isolates for tagging and then cloning of several avirulence genes, such as AvrPi15, AvrPi7, AvrPik, AvrPikm, AvrPik-p, and AvrPib (6,15; Q. Pan, unpublished data).

In summary, MAT1-1 and MAT1-2 are maintained in $M$. oryzae in southwestern China (especially Yunnan) that contain many highly fertile and hermaphroditic isolates. Although sexual reproduction has not been observed under field conditions in rice, $M$. oryzae-favorable environmental conditions do exist in which this could and thus probably does occur $(12,16)$.

\section{ACKNOWLEDGMENTS}

We thank E. Roumen for providing tester isolates TH12 and TH16; and Y. Zhu (Yunnan Agricultural University), X. Zheng (Nanjing Agricultural University), Z. Wang (Fujian Agricultural and Forestry University), and J. Yuan (Agricultural Academy Sciences of Guizhou Province) for the other M. oryzae isolates used in this study. Financial support was provided by the National Industry

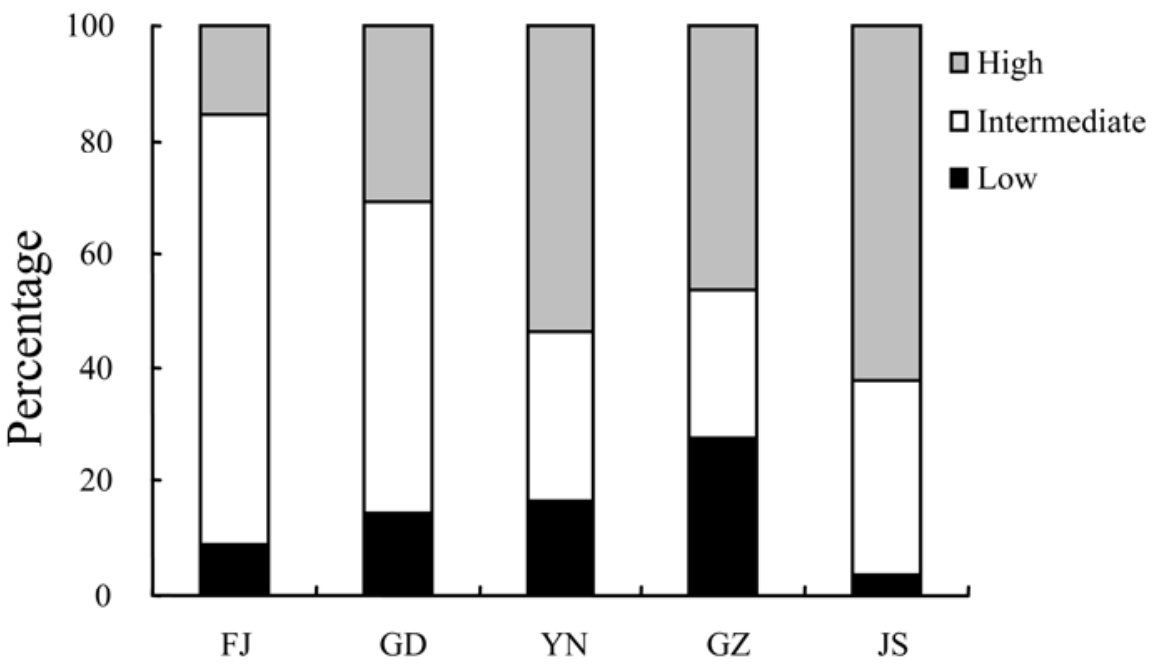

Location

Fig. 4. Proportion of fertile isolates in each fertility class, based on perithecium density $(<10,10$ to 19 , $\geq 20$ perithecia/ $\mathrm{cm}^{2}$ refer to the poorly, intermediate, and highly fertile classes, respectively). FJ: Fujian, 70 isolates; GD: Guangdong, 139 isolates; YN: Yunnan, 91 isolates; GZ: Guizhou, 69 isolates; JS: Jiangsu, 74 isolates.
Research Project (200803008) and the National 973 project (2006CB/1002006).

\section{LITERATURE CITED}

1. Cai, J., Wang, L., and Pan, Q. 2005. Mating type and fertility of the rice blast fungus population derived from Guangdong province, China. Sci. Agric. Sin. 38:837-842. (In Chinese with English abstract)

2. Consolo, V. F., Cordo, C. A., and Salerno, G. L. 2005. Mating-type distribution and fertility status in Magnaporthe grisea populations from Argentina. Mycopathologia 160:285-290.

3. Couch, B. C., and Kohn, L. M. 2002. A multilocus gene genealogy concordant with host preference indicates segregation of a new species, Magnaporthe oryzae, from M. grisea. Mycologia 94:683-693.

4. Dai, L., Xiong, J., Wen, G., Chen, Y., Ye, C., Nagamine, T., Sunohara, Y., and Tomita, K. 1995. Further information on the genetic variation of indigenous rice varieties in Yunnan province, China. Breed. Sci. 45:397-399.

5. Dioh, W., Tharreau, D., Notteghem, J. L., Orbach, M., and Lebrun, M. H. 2000. Mapping of avirulence genes in the rice blast fungus, Magnaporthe grisea, with RFLP and RAPD markers. Mol. Plant-Microbe Interact. 13:217227.

6. Feng, S., Wang, L., Ma, J., Lin, F., and Pan, Q. 2007. Genetic and physical mapping of $\mathrm{AvrPi7}$, a novel avirulence gene of Magnaporthe oryzae using physical position-ready markers. Chin. Sci. Bull. 52:903-911.

7. Hayashi, N., Li, C., Li, J., Iwano, M., Naito, H., Yoshino, R., and Kato, H. 1997. Distribution of fertile Magnaporthe grisea fungus pathogenic to rice in Yunnan Province, China. Ann. Phytopathol. Soc. Jpn. 63:316-323.

8. Hayashi, N., Li, C., Li, J., and Naito, H. 1997. In vitro production on rice plants of perithecia of Magnaporthe grisea from Yunnan, China. Mycol. Res. 101:1308-1310.

9. Itoi, S., Mishima, T., Arase, S., and Nozu, M. 1983. Mating behavior of Japanese isolates of Pyricularia oryzae. Phytopathology 73:155158.

10. Kato, H., and Yamaguchi, T. 1982. The perfect state of Pyricularia oryzae Cav. from rice plants in culture. Ann. Phytopathol. Soc. Jpn. 48:607-612.

11. Kolmer, J. A., and Ellingboe, A. H. 1988. Genetic relationships and virulence to rice in Magnaporthe grisea. Can. J. Bot. 66:891-897.

12. Kumer, J., Nelson, R. J., and Zeigler, R. S. 1999. Population structure and dynamics of Magnaporthe grisea in the Indian Himalayas. Genetics 152:971-984.

13. Leslie, J. F., and Klein, K. K. 1996. Female fertility and mating type effects on effective population size and evolution in filamentous fungi. Genetics 144:557-567.

14. Luo, C., Yin, L., Koyanagi, S., Farman, M. L., Kusaba, M., and Yaegashi, H. 2005. Genetic mapping and chromosomal assignment of Magnaporthe oryzae avirulence genes AvrPik, AvrPiz, and AvrPiz-t controlling cultivar specificity on rice. Phytopathology 95:640-647.

15. Ma, J., Wang, L., Feng, S., Lin, F., Xiao, Y., and Pan, Q. 2006. Identification and fine mapping of AvrPi15, a novel avirulence gene of Magnaporthe grisea. Theor. Appl. Genet. 113:875-883.

16. Mekwatanakarn, P., Kositratana, W., Phromraksa, T., and Zeigler, R. S. 1999. Sexually fertile Magnaporthe grisea rice pathogens in Thailand. Plant Dis. 83:939-943.

17. Noguchi, M., Yasuda, N., and Fujita, Y. 2006. Evidence of genetic exchange by parasexual recombination and genetic analysis of pathogenicity and mating type of parasexual recombinants in rice blast fungus, Magnaporthe oryzae. Phytopathology 96:746-750.

18. Notteghem, J. L., and Silué, D. 1992. Distribu- 
tion of the mating type alleles in Magnaporthe grisea populations pathogenic on rice. Phytopathology 82:421-424.

19. Notteghem, J. L., Tharreau, D., Silué, D., and Roumen, E. 1994. Present knowledge of rice resistance genetics and strategies for Magnaporthe grisea pathogenicity and avirulence gene analysis. Pages 155-165 in: Rice Blast Disease. R. S. Zeigler, S. A. Leong, and P. S. Teng, eds. CAB International, Wallingford, Oxon, UK.

20. Pushpavathi, S., Thakur, R. P., and Chandrashekara Rao, K. Fertility and mating type frequency in Indian isolates of Sclerospora graminicola, the downy mildew pathogen of pearl millet. Plan Dis. 90:211-214.

21. Sommerhalder, R. J., McDonald, B. A., and
Zhan, J. 2006. The frequencies and spatial distribution of mating types in Stagonospora nodorum are consistent with recurring sexual reproduction. Phytopathology 96:234-239.

22. Tredway, L. P., Stevenson, K. L., and Burpee, L. L. 2003. Mating type distribution and fertility status in Magnaporthe grisea populations from turfgrasses in Georgia. Plant Dis. 87:435441.

23. Urashima, A. S., Igarashi, S., and Kato, H. 1993. Host range, mating type, and fertility of Pyricularia grisea from wheat in Brazil. Phytopathology 77:1211-1216.

24. Valent, B., and Chumley, F. G. 1991. Molecular genetic analysis of the rice blast fungus, Magnaporthe grisea. Annu. Rev. Phytopathol. 29:443-467.
25. Yaegashi, H., and Nishihara, N. 1976. Production of the perfect stage in Pyricularia from cereals and grasses. Ann. Phytopathol. Soc. Jpn. 42:511-515.

26. Yasuda, N, Noguchi, M., and Fijita, Y. 2005. Identification of an avirulence gene in the fungus Magnaporthe grisea corresponding to resistance gene at the Pik locus. Phytopathology 95:768-772.

27. Zeigler, R. S. 1998. Recombination in Magnaporthe grisea. Annu. Rev. Phytopathol. 36:249-275.

28. Zhu, Y., Chen, H., Fan, J., Wang, Y., Li, Y., Chen, J., Fan, J., Yang, S., Hu, L., Leung, H. Mew, T. W., Teng, P. S., Wang, Z., and Mundt, C. 2000. Genetic diversity and disease control in rice. Nature 406:718-722. 